\title{
Speed control of robust position sensor less PMBLDC motor by Fuzzy controller
}

\author{
Shravani Sattu ${ }^{1}$, Vinay Kumar Awaar ${ }^{1 *}$, and Praveen Jugge ${ }^{1}$ \\ ${ }^{1}$ Department of EEE, GRIET, Hyderabad, Telangana, India
}

\begin{abstract}
The aim of this work is to present a novel mechanical sensor-less control approach for permanent magnet brushless DC (PMBLDC) motors based on speed independent $\mathrm{H}$ functions. The starting of the motor and driving it from a low speed to the rated speed are two significant problems in sensor-less operation of PMBLDC motor drives. The commutation moment is determined using a speed-independent $\mathrm{H}$ function. Since the commutation instant is speed independent, it may be anticipated even at low speeds. The $\mathrm{H}$ function is entirely dependent online voltages, which simplifies and reduces the cost of the whole system. The suggested approach eliminates the need for analogue filtering and phase correction. Digital low pass filters and digital phase compensators are used to remove commutation ripple from line voltages. This results in a system that is small, simple to use, and cost effective. This economical option is ideal for low-cost equipment such as fans, air conditioners, refrigerators, and vacuum cleaners. The suggested method is implemented using a fuzzy controller to demonstrate the proposed sensor-less technique's efficacy for wide speed variations.
\end{abstract}

\section{Introduction}

In the last three decades the reduction of PMBLDCM functioning has always been an appealing study topic for academia (Permanent Magnet Brushless DC Motor). As the use of HallEffect electronic sensors is essential for rotor position assessments, due to the extra weather and circuitry a system is expensive and complex. A halleffect sensor is also sensitive and successful with restricted temperature fluctuations. Therefore, the usage of a PMBLDC engine in both industrial and residential applications was restricted for those reasons. Many efforts have been made in a literature to overcome these deficiencies. The simple technique to estimate PMBLDCM's location is a strategy for EMF sensing [1-5]. A sensor in connection to a virtual soil of a zero-phase transmission is used to calculate the switching timings of this approach. However, a phase voltage zero-crossing instant is not a genuine moment. A null-crossing instant must be modified in phase to create the correct switching instant. A zero passing of line voltages is the extra approach in the category. A line voltage might be twice as - phase back veterinarian EMF. [6-7] has been demonstrated to offer the switch-up immediate to zero EMF return phase. The low-speed performance of these methods is a major disadvantage. The efficiencies of a technique above are not excellent because a low-speed EMF stage return is too low to be accurately sensed. A perceived back emf from a quiet phase is integrated into another technique [8] and [9] until a threshold is reached. Integration begins with a null crossing and returns a threshold value for the back phasing emf. It is established that a threshold value will nearly overlap with an exact time of switching. This method also shows inefficiency and low efficiency at lower speeds. A novel, technologically less sensor method has been outlined in the three-phase balanced star [4] and [10], which connects the three-star resistor with the neutral point, n. A voltage of three times the frequency of a trapezoidal rear emf between two neutral locations (Vnn'). This approach is hence termed as an EMF detection technique as a third harmonic back. A list of VNNs is added below and a decision is made at the time of switching. AVnnTes zero crossing was changed by 300 phases from an exact point of change, yet the integration corresponds precisely with a moment of switching. A neutral point of an engine is necessary for this operation. Another technique [11] for immediate switching measures the current of a quiet phase freewheeling diode. Because of inductive engine winding, freewheels through the diode are present in a quiet phase. A switching moment is picked by monitoring this current. There are six current measuring circuits that make this technology a complicated, costly, and large gadget. [1] for very

\footnotetext{
*Corresponding author: vinaykumaar.a@gmail.com
} 
accurate rapid predictions of switching has created a fast, autonomous function. A sensor operates less than a high-speed PMBLDC engine, as a switching moment is independent from a speed, the authors demonstrated. The control is broken down into many kinds of work. Information about a prior mode is necessary to determine a current operating mode. Three separate $\mathrm{H}$ functions are developed for quick switching using a suggested approach. These $\mathrm{H}$ functions are determined by detecting line voltages; a neutral point is thus not required. As the PMBLDCM consists of balanced three-phase windings, a third one and two others are calculated using two-line voltages solely. The given approach must be utilised from zero to full speed due to the independence of the speed of the function $\mathrm{H}$. Three $\mathrm{H}$ functions control each phase individually. The real value of a $\mathrm{H}$ function depends completely on a three-phase voltage sources instant and pulse of the inverter. Using this technique, a PMBLDC engine with Hall sensors is increased dependability.

The main advantages of a proposed technique to control are to information from previous states will be needed if a VSI pulse is immediately switched and changed. For sensing and computing $\mathrm{H}-$ functions, just 2-line voltages are necessary. No filter or trim circuit equivalent is necessary. This reduces the value of a system. No large computation is necessary for this technique. Consequently, a DSP implementation is easy on the low cost.

There are essentially differences in the strategy and methods described [1]. The rotor location should be split into six intervals, utilising the proposed approach. For instant switching, each period is analysed individually. EMF form and phase streams are utilised to simplify the H-function throughout each interval analysis. Kim et.al [1] designed a single switching function for all rotors, however, to determine the moment of switching, Kim et.al[1] created a G-Function. G-function frequencies are twice as many as phase currents and phase line shapes, whereas the H-function frequency is equivalent to the phase currents frequency and the line voltage.H-functions are based on changes in line voltage only, whereas an approach is based on threeline currents for Kim, etc.[1]. The current magnitude of the voltage line simply requires an estimated rotor position at any time using the approach described. A Kim et.al method[1] does require, however, DC connection voltage, and three phase streams and phase current data at that point in time. For both positive and negative phase fluctuations, a $G$ function waveform is the same since the $G$ function frequency is a two-fold phase. Thus, data from a previous current phase is required to establish whether a G-function wave shape is the same for the positive as well as negative phase currents. Therefore, a time to switch for information about an instant previous phase must be determined. A phase delay for a waveform sensing was addressed in the proposed technique.

\section{PMBLDC motor proposed sensor less technique}

An extensive literature on mathematical modelling of the PMBLDC engine was discussed [21-23]. The PMBLDC motor focuses on its stator in $1200 \mathrm{VSI}$ mode and its permanent magnets in a rotor. Therefore, a current always begins with a single phase, leaves the second and third phases open. Therefore, only two phases are operating at any moment. Phase 'a' in neutral phase of tension equation

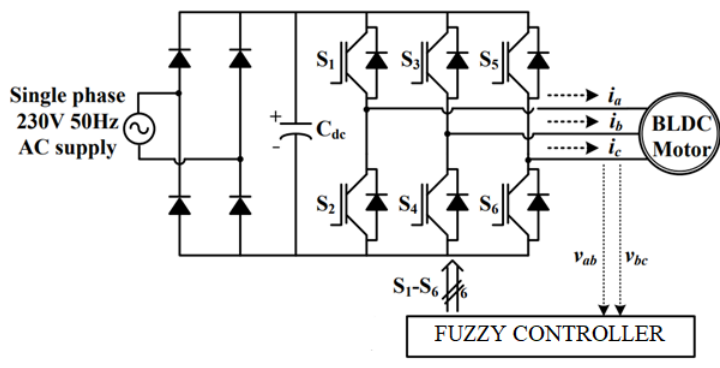

Fig. 1. Proposed BLDC motor Block diagram

\subsection{Mathematical modelling of PMBLDC Motor}

An extensive literature on a mathematical modelling of a PMBLDC engine has been discussed[20]. The PMBLDC motor focuses on its stator in $1200 \mathrm{VSI}$ mode and its permanent magnets in a rotor. Therefore, a current always begins with a single phase, leaves the second and third phases open. Therefore, only two phases are operating at any moment. Phase 'a' is a neutral phase of tension equation

$$
V_{a n}=i_{a} R+L \frac{d i_{a}}{d t}+\frac{d\left(\lambda_{a f}(\theta)\right)}{d t}
$$

Whereas $V_{a}, V_{b}$ and $V_{c}$ are line voltages $i_{a}, i_{b}$ and $i_{c}$ are line currents, where the $\mathrm{R}$ is the phase resistance, $\mathrm{L}$ is the phase inductance. A changing rotor flow rate which connects a phase winding is called a induced EMF. (2) is therefore modified,

$$
V_{a n}=i_{a} R+L \frac{d i_{a}}{d t}+E_{a n}
$$

Where, $E_{a}, E_{b}$ and $E_{c}$ are induced rear EMF. Likewise, for other phases voltage equations are given,

$$
\begin{aligned}
& V_{b n}=i_{b} R+L \frac{d i_{b}}{d t}+E_{b n} \\
& V_{c n}=i_{c} R+L \frac{d i_{c}}{d t}+E_{c n}
\end{aligned}
$$


Therefore, line voltages are expressed as neutral point not accessible.,

$$
\begin{aligned}
V_{a b}=V_{a n}-V_{b n}= & i_{a} R+L \frac{d i_{a}}{d t}+E_{a n}-\left(i_{b} R+\right. \\
& \left.L \frac{d i_{b}}{d t}+E_{b n}\right)
\end{aligned}
$$

$V_{a b}=\left(i_{a}-i_{b}\right) R+L \frac{d\left(\left(i_{a}-i_{b}\right)\right)}{d t}+E_{a n}-E_{b n}$

As follows,

$V_{b c}=\left(i_{b}-i_{c}\right) R+L \frac{d\left(\left(i_{b}-i_{c}\right)\right)}{d t}+E_{b n}-E_{c n}$

$V_{c a}=\left(i_{c}-i_{a}\right) R+L \frac{d\left(\left(i_{c}-i_{a}\right)\right)}{d t}+E_{c n}-E_{a n}$

Sensing voltage at motor terminals can be accessible by these line voltages. Vabc and Vbc vary, a difference

$$
\begin{aligned}
V_{a b b c}= & v_{a b}-V_{b c}=\left[\left(i_{a}-i_{b}\right) R+L \frac{d\left(\left(i_{a}-i_{b}\right)\right)}{d t}+\right. \\
& \left.E_{a n}-E_{b n}\right]-\left[\left(i_{b}-i_{c}\right) R+L \frac{d\left(\left(i_{b}-i_{c}\right)\right)}{d t}+\right. \\
& \left.E_{b n}-E_{c n}\right] \\
V_{a b b c}= & \left(i_{a}+i_{b}-2 i_{b}\right) R+L \frac{d\left(i_{a}+i_{c}-2 i_{b}\right)}{d t}+\left(E_{a n}+\right. \\
& \left.E_{c n}-2 E_{b n}\right)
\end{aligned}
$$

Also, $V_{a b}, V_{b c}$ and $V_{a c}$ are a following differences,

$$
\begin{aligned}
V_{b b c a}= & \left(i_{b}+i_{a}-2 i_{c}\right) R+L \frac{d\left(i_{b}+i_{a}-2 i_{c}\right)}{d t}+\left(E_{b n}+\right. \\
& \left.E_{a n}-2 E_{c n}\right)
\end{aligned}
$$$$
V_{c a a b}=\left(i_{c}+i_{b}-2 i_{a}\right) R+L \frac{d\left(i_{c}+i_{b}-2 i_{a}\right)}{d t}+\left(E_{c n}+\right.
$$$$
\left.E_{b n}-2 E_{a n}\right)
$$

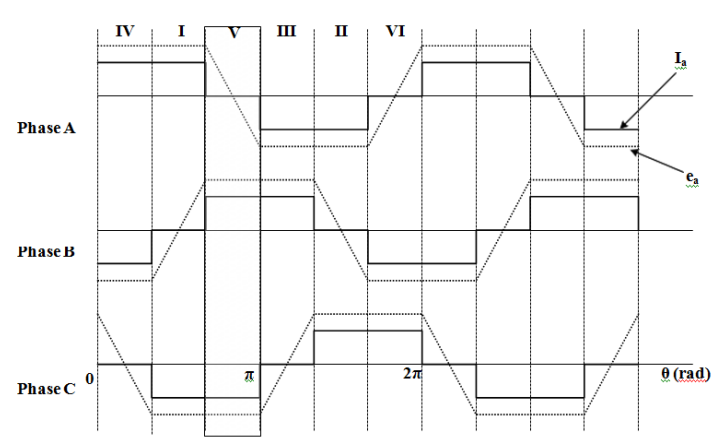

Fig. 2. Three phase back emf and phase current of PMBLDC motor.

\subsection{Mathematical modelling of proposed method}

Fig.2 shows a induced emf back and a current of every winding step. These shapes are shown in comparison to a rotor's position. A figure indicates that a current for winding depends on where a rotor is located. For motor excitement, a rotor position is consequently unavoidable. Every roll is excited for 1200 . This is why a figure is split by rotor position in six different intervals. There are just two phases, whereas a third one is still available. Take interval I into account. Phase ' $a$ ' is now entered and phase 'c is returned. Phase "a" stage is constant in backdrop and positive, however phase "c" stage is negative in background. Phase ' $c$ ' is also entered during interval II and Phase ' $a$ ' is returned in present phase, and it is zero in Phase 'b.'

For intervals I and II,

$i_{a}=-i_{c}, i_{b}=0$ and $E_{a n}=-E_{c n}$

Hence, $(10-12)$ are modified as,

$$
\begin{aligned}
V_{a b b c}=- & 2 E_{b n} V_{c a a b}=-3 i_{a} R-3 L \frac{d i_{a}}{d t}-3 E_{a n}+ \\
& E_{c n}
\end{aligned}
$$

$V_{a b b c}=3 i_{a} R+L \frac{d i_{a}}{d t}+3 E_{a n}+E_{c n}$

From (13 - 15),

$\frac{3 E_{a n}+E_{c n}}{-E_{c n}}=\frac{V_{a b b c}-3 i_{a} R-3 L \frac{d i_{n}}{d t}}{V_{b c c a}}$

$\frac{3 E_{a n}}{E_{c n}}=\frac{6 i_{a} R+62 \frac{d i_{n}}{d t}-2 V_{a b b c}-V_{b c c a}}{V_{b c c a}}$

As follows, for intervals $\mathrm{V}$ and $\mathrm{VI}$,

$i_{b}=-i_{c}, i_{a}=0$, and $E_{b n}=-E_{c n}$

Hence, (16 - 18) are rewritten as,

$V_{c a a b}=-2 E_{a n}$

$V_{a b b c}=-3 i_{b} R-3 L \frac{d i_{b}}{d t}-3 E_{b n}+E_{a n}$

$V_{b c c a}=3 i_{b} R+3 L \frac{d i_{b}}{d t}+3 E_{b n}+E_{a n}$

$\frac{3 E_{b n}+E_{a n}}{-2 E_{a n}}=\frac{V_{b c c a}-3 i_{a} R-3 L \frac{d i_{h}}{d t}}{V_{c a a b}}$

$\frac{3 E_{b n}}{E_{a n}}=\frac{6 i_{b} R+6 L \frac{d i_{h}}{d t}-2 V_{b c c a}-V_{c a a b}}{V_{c a a b}}$

As, $i_{b}=-i_{c}, i_{a}=0$, and $E_{b n}=-E_{c n}$,

$\frac{-3 E_{c n}}{E_{a n}}=\frac{-6 i_{c} R-62 \frac{d i_{c}}{d t}+-2 V_{b c c a}-V_{c a a b}}{V_{c a a b}}$
$\frac{-3 E_{c n}}{E_{a n}}=\frac{6 i_{c} R+62 \frac{d i_{c}}{d t}-+2 V_{b c c a}-V_{c a a b}}{V_{c a a b}}$ 
The moment of any phase change is approached (17, 24 and 31). However, these signals are twice as often about a $\mathrm{Y}$-axis as phase voltage and current. To decide for an instant switch, these functions require information on an earlier switching instant. To make a switching immediately independent of past states, the further term is introduced to its $\mathrm{H}$ functions. In these three signals a following are further modified:

$H_{a b}=\operatorname{sign}\left(V_{a b b c}\right) \frac{6 i_{a} R+62 \frac{d i_{a}}{d t}--2 V_{b c c a}-V_{a b b c}}{V_{a b b c}}$

$H_{b c}=\operatorname{sign}\left(V_{b c c a}\right) \frac{6 i_{b} R+62 \frac{d i_{a}}{d t}+2 V_{a b b c}+V_{b c c a}}{V_{b c c a}}$

$H_{c a}=\operatorname{sign}\left(V_{c a a b}\right) \frac{6 i_{c} R+62 \frac{d i_{c}}{d t}+2 V_{b c c a}+V_{c a a b}}{V_{c a a b}}$

$6 i_{a} R+6 L \frac{d i_{a}}{d t}<<\left(2 V_{b c c a}+V_{a b b c}\right)$

$H_{a}, H_{b}, H_{c}$ are $\mathrm{H}$ functions

This is clearly illustrated in Fig. 3. This distinction. Here you can observe a contrast between two sentences at 1500 to 3000 . From this image it can simply be seen that a first period is near zero and that there are high points at a moment of switching. $\mathrm{AH}$ functions reflect these peaks, which could result in wrong switching. To detect the suitable switching instant, a first term of a $\mathrm{H}$ function is disregarded. Above assumptions work well with the wide variety of speed changes as $\mathrm{H}$ functions are velocity independent. However, an assumption depends on a current of phases. A waveform of $\mathrm{H}$ depends on a current of a phase. Therefore, with changes in phase currents, a form of a $\mathrm{H}$ functions varies. In Fig. 15 this discrepancy is describable (a-b).

$$
\text { As } 6 i_{a} R+6 L \frac{d i_{a}}{d t}<<\left(2 V_{b c c a}+V_{a b b c}\right)
$$

therefore, (32) is approximated as,

$H_{a b}=\operatorname{sign}\left(V_{a b b c}\right) \frac{-2 V_{b c c a}-V_{a b b c}}{V_{a b b c}}$

As follows, (33-34) can be modified as,

$$
\begin{aligned}
& H_{b c}=\operatorname{sign}\left(V_{b c c a}\right) \frac{2 V_{a b b c}+V_{b c c a}}{V_{b c c a}} \\
& H_{c a}=\operatorname{sign}\left(V_{c a a b}\right) \frac{2 V_{b c c a}+V_{c a a b}}{V_{c a a b}}
\end{aligned}
$$
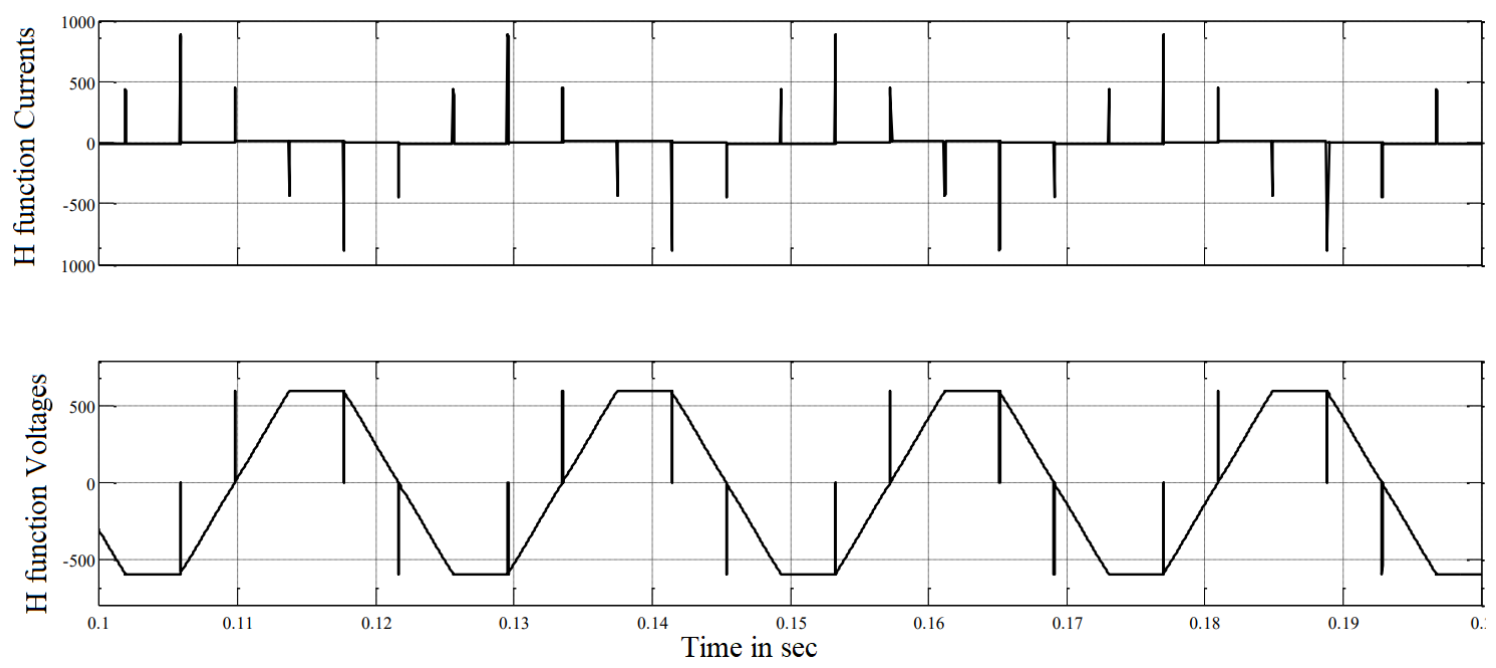

Fig. 3. $6 \mathrm{iaRa}+6 \mathrm{La}(\mathrm{dia} / \mathrm{dt})$ comparison with $2 \mathrm{vbcca}+\mathrm{vabbc}$ has shown that a first term was considerably low in $1300 \mathrm{rpm}$ compared to a second term

There is little supposition for a following if a $\mathrm{H}$ functions are derived and summarised. Permeability of a core material is not known. Air corresponds to a magnets' permittivity. Phase induction thus depends on a rotor position. Motor winding phase is considered balanced and identical. Leakage flow and fringe impact are neglected.

\section{BLDC speed controllers}

Some rules are accessible now, such as a PI, Fuzzy or combination: Fuzzy-Genetic Algorithms, FuzzyNeural Networks, Fuzzy-Swarm, Fuzzy-Ants Colony. There are now the couple of these controls.
However, this page covers a discussion on a PI and a Fuzzy Logic Controller as beneath. Proportional Integral (PI) Speed Controller is electronic control loop feedback mechanism. PI Controller finds its applications in various industrial processes when a controller seeks to determine an error between a process variable and a reference point. An algorithm includes the process-changing corrective action for calculating and producing. PI controller has two separate modes, proportional and integral, as a name suggests. A reaction to that error is calculated in proportion, while a recent reaction-based error is derived in integrated mode [6]. A simple design and ease of utilisation of PI controls in industry are often used. Fig. 4. displays a controller PMBLDC based 
drive. Fig.4. A drive includes a speed control, a refer Strom generator, a control on a PWM current, a position sensor, an engine and a IGBT inverter (VSI).

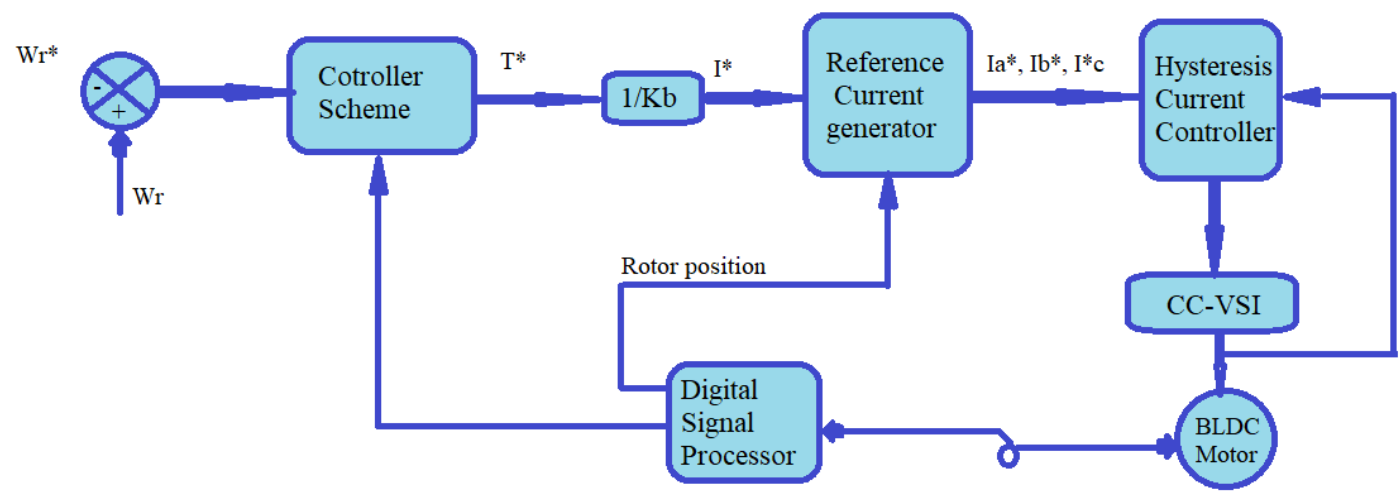

Fig 4. BLDC Controller Scheme Diagram.

The engine speed shall be compared with reference value and PI controller shall treat a speed error. Fig4 shows speed control algorithm of a BLDC engine. This flowchart generalises a speed loop of a conventional BLDC motor to any type of driver [16].

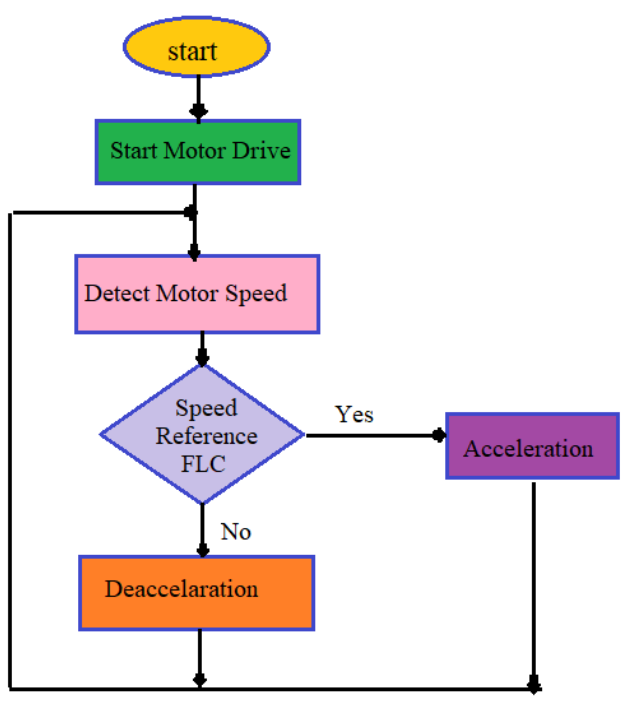

Fig. 5. Flow chart of a FLC

The above shown diagram represents the flowchart of Fuzzy Logic controller. The actual controlling scheme is represented briefly.

The Fuzzy logic controller facilitates modelling of non-linear systems (FLC). General based on a mathematical model of a plant; a conventional control system is often sophisticated. On a one hand, FLC expresses an operational law on language words rather than mathematical equations [11]. Many systems, including complex mathematical equations, were sometimes found to be too complex to efficiently module.

Consequently, in these instances typical procedures are impractical. Fuzzy language logics are now the realistic and uncomplicated technique to develop and implement a system's operative characteristics [15]. BLDC generic block diagram can be replaced by controls as shown in Figure 2 as requested by each other controller. Every control mechanism that is suitable for a maintenance and regulation of a BLDC motor can be used to systems such as PI, or Fuzzy Logical Controller. Next section deals with a simulated BLDC engine link with PI and Fuzzy logical controller and discusses a result of a simulations in section 4 .

\section{Simulation results}

A Simulink model is designed to serve the permanent BLDC motor with an IP controller in the Simulink tool in MATLAB. Simulink model consists of three-phase supply inverter and BLDC engine. To control a motor speed, the PI control system is attached to a model. A Simulink model is the $10.5 \mathrm{sec}$ runtime and for different parameter values as shown above a system is simulated.

Fig. 1 Displays Simulink model for a PI controller engine of a BLDC. Models are an engine speed control model with a PI controller MATLAB Simulink. A model includes the power supply inverter and the BLDC motor. A speed of $3000 \mathrm{rpm}$ of the motor is reference value. Here, BLDC engine meets an operational concept outlined. 


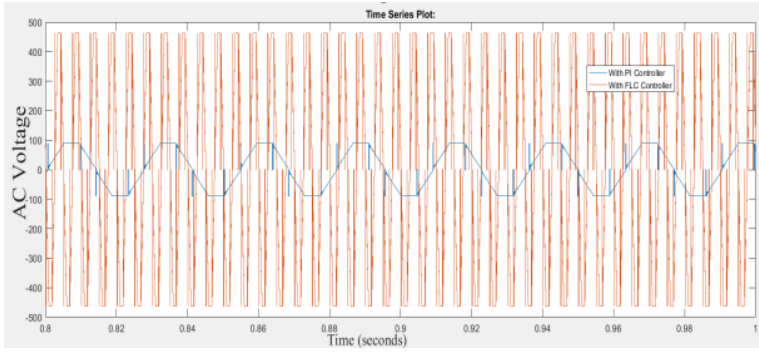

Fig 6. AC Voltage of the inverter with PI and Fuzzy controller

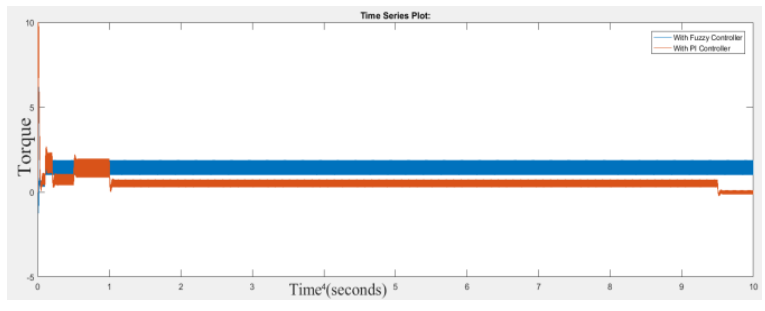

Fig 7. BLDC electromagnetic torque with PI and Fuzzy controller

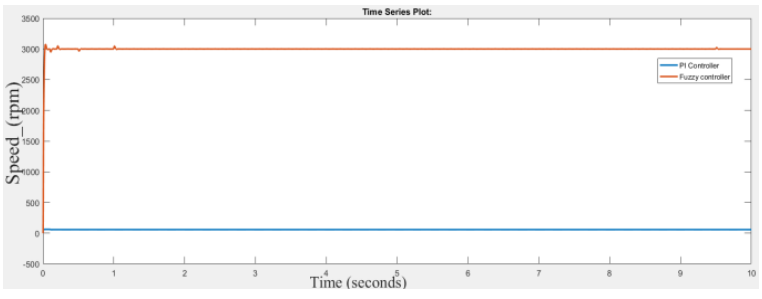

Fig 8. BLDC motor speed currents with PI and Fuzzy controller

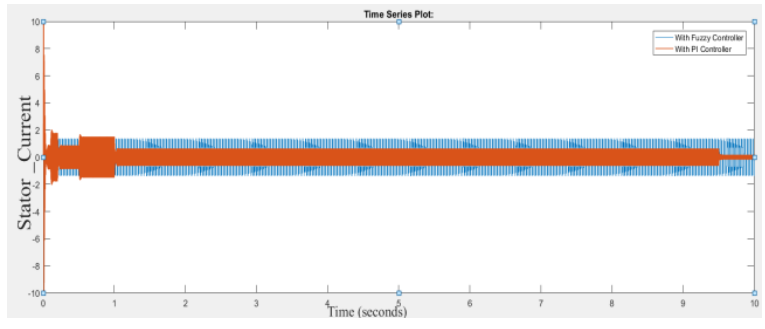

Fig 9. BLDC stator currents with PI and Fuzzy controller

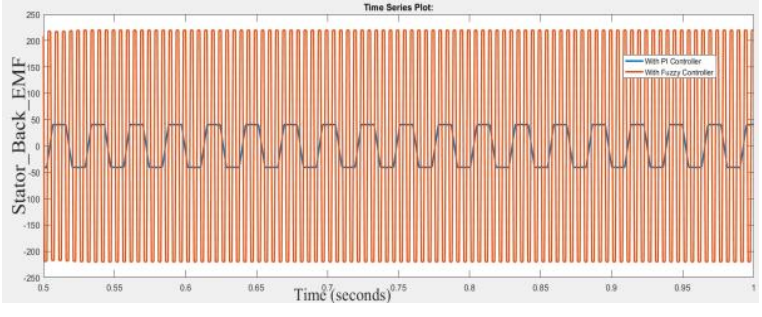

Fig 10. BLDC stator back emf with PI and Fuzzy controller

Fig10.Three-phase reverse-voltage power supply and the BLDC engine are a part of a Simulink version. FLC is intended using input and output parameters of fuzzy controller; it has the membership functions of 7 rule sets and has three-way functions of a type it employs.

Fuzzy Controller Training comprises of:
7 inputs and outputs 1. 1. 1, fuzzification of inputs across a cosmos of continual speech, Mamdani's connotation with 'min', For defuzzification a 'centre' approach is employed. The straight-line equation is utilised for a production of fuzzy sets for easy computations. This triangle curve can be shown as

$\mu_{A}(x)=\max \left(\min \left(\frac{x-a}{b-a}, \frac{c-x}{c-b}\right)\right.$

where

$\mu_{A}(x)$ is defuzzification a, b, c is b-a c-b scalar parameters

FLC inputs are error(s), an error(s), and an active current component. Aresult is altered. Table I defines inputs and outputs ofFLC. E' and 'cue' are changed from continuous data to fluctuating data by using seven membership functions. As a construction is basic, a membership functions are triangular. All seven members comprised a names 'NB'-negative large' and 'NM'-negative medium, 'NS'-negative little, 'ZE'-zero, 'PS-positive little,' 'PM'-positive medium' and 'PB'- positive large. A spectrum of ' $E$ 'and 'CE' values is based on discrimination against membership functions. An output of a fluid logical controller is calculated using Table I. For each of a inputs, a outcome will be 49 members. An outcome of a relevant input values is displayed in Table I. Since FLC is carefully built and does not have the system mathematical modelling, a above input and output variables of the combination of seven are translated by the method called Fuzzification into a language variable. Error sets and changes to errors are linked to system rules. system rules are generated. A system rule basis as shown in Table I is known as these regulations. With fugitive knowledge, the deduction is produced that gives output to a system. Based on a abovementioned table and simulation, the BLDC model has been developed using an FLC in a MATLAB Simulink tool.

Table 1: Rule base functions

\begin{tabular}{|c|c|c|c|c|c|c|c|}
\hline E/CE & NB & NM & NS & ZE & PS & PM & PB \\
\hline NB & NB & NB & NB & NB & NM & NS & ZE \\
\hline NM & NB & NB & NB & NM & NS & ZE & PS \\
\hline NS & NB & NB & NM & NS & ZE & PS & PM \\
\hline ZE & NB & NM & NS & ZE & PS & PM & PB \\
\hline PS & NM & NS & ZE & PS & PM & PB & PB \\
\hline PM & NS & ZE & PS & PM & PB & PB & PB \\
\hline PB & ZE & PS & PM & PB & PB & PB & PB \\
\hline
\end{tabular}

PMBLDC Simulink model of FLC model, and pretime results were shown. Fig.4 A results consists of an FLC speed, torque, and current characteristics curve of the BLDC motor. This is contrasted with a finding of a PI controller. The PI and FLC for speed control of a BLDC engine, respectively. For both controllers parameters are listed in Table 1. API controller settlement time is $1 \mathrm{~s}$, while Fuzzy PI controls have the settlement time of $0.02 \mathrm{~s}$. API 
controller and a FLC response speed curve provide other performance characteristics. Table IV shows a FLC performance being higher than that of a PI as a predicted FLC is unusually low by $0.02 \mathrm{~s}$. And there is enhanced stability without oscillations or less transients.

\section{Conclusions}

This study exhibits a speed management by both the PI controller and Fuzzy Logic Controller of the permanent Magnet BLDC engine. In summary, it offers BLDC's performance research. In an MA TLAB Semolina tool for the BLDC speed control, a compare analysis has been talked between the PI control unit and the Fuzzy logic controller. The results produced separately by both controllers are compared and analysed to assess speed controllers' performance. After the comparison, one result was that the BLDC speed control using a Fuzzy logic controller is more efficient. In future it may be a task for a recommended speed controller to add a current control function to keep a current within the stated speed range. This will improve a start-up motor current, minimise motor current waves and increase the overall motor torque efficiency. A speed and torque of current motor values via fast changes will also be a current control method.

\section{References}

1. B. Tibor, V. Fedak and F. Durovsky, TEEE (2011).

2. W. Hong, W. Lee and B. K. Lee, IEMDC 13, (2007)

3. Murat Cahin, H . I Bulbul, Zafer Esen, U. Yavanoglu and I. Colak, POWERENG, Istanbul, (2013).

4. H. K. SamithaRansara and U. K. Madawala, TEEE, Cape Town, (2013).

5. L. Zhao, X. Zhang and JunhongJi, ICMA), TEEE, Beijing, (2015).

6. M.Harith, K. P. Remya, Kalady AS ET, and S. Gomathy. Intl. Res. JE of Eng. and Tech., 2,875, (2015).

7. Z. Ibrahim and E. Levi, IEEE Trans. on Ind. Appl., 38, 1210(2002).

8. M. Cheng, Qiang Sun and E. Zhou, IEEE Tran. on Ind. Elec., 53, 814, (2006).

9. Sant and K. R. Rajagopal, IEEE Tran. on Mag., 45, 4672(2009).

10. T. C. Siong, B. Ismail, S. F. Siraj, M. F. N. Tajuddin, N. S. Jamoshid and M. F. Mohammed, SCOReD, (2010).

11. G. Prasad, N. SreeRamya, P. V. N. Prasad, and G. Tulasi Ram Das. Proceedings of IJiTEE 1, no. 5, 2278(2012).

12. Tashakori and M. Ektesabi, ICIEA, Melbourne, VIC, (2013).
13. H. S. EI-Sayed, F. M. EI-Khouly, M. M. Khater and the M. Osheiba, ICEE, (2007)

14. N. Parhizkar, M. Shafiei and M. B. Kouhshahi, URKE, Bali, (2011).

15. S. P. And S. ISGT, IEEE PES, Kerala, (2011).

16. M. Ahmed, Amr Ali-Eldin, Mohamed S. Elksasy, and Faiz F. Areed. Intl. J of Comp. Appl. 10, 29(2015).

17. Awaar, Vinay Kumar, Praveen Jugge, S. Tara Kalyani, and Someshwara Thota. IEEE SeFET, (2021).

18. Awaar, V.K., Jugge, P. \& Tara Kalyani, S. J Control AutomElectrSyst 30: 601, (2019).

19. B. J. Varghese, P. B. Bobba and M. Kavitha, 2016 IEEE PIICON, (2016).

20. Kavitha Merugu., Prasad Dinkar, Bobba Phaneendra Babu, IET Ele. Power Appl., 13, 1184, (2019).

21. M. Kavitha, P. B. Bobba and D. Prasad, IEEE ICPS, (2016).

22. Ganesh, R., Subbiah, R., Chandrasekaran, K. Mat. Tod. Proc., 2, 1441 (2015).

23. Usha Kumari, Ch., Jeevan Prasad, S., Mounika, G. Proc. of ICCMC 2019, (2019). 